\title{
Defective Platelet Activation and Bleeding Complications upon Cholestasis in Mice
}

\author{
Nina Sarah Gowerta,b Meike Klier ${ }^{a, b}$ Maria Reich ${ }^{c}$ Friedrich Reusswiga,b \\ Lili Donnera,b Verena Keitel ${ }^{\mathrm{c}}$ Dieter Häussinger ${ }^{c}$ Margitta Elvers ${ }^{\mathrm{a}, \mathrm{b}}$ \\ aDepartment of Clinical and Experimental Hemostasis, Hemotherapy and Transfusion Medicine, \\ Heinrich-Heine-University Düsseldorf, Düsseldorf, ${ }^{b}$ Department of Vascular and Endovascular Surgery, \\ Heinrich-Heine-University Düsseldorf, Düsseldorf, 'Clinic for Gastroenterology, Hepatology and \\ Infectious Diseases, Heinrich-Heine-University Düsseldorf, Düsseldorf, Germany
}

\section{Key Words}

Platelets - Cholestatic liver disease - Hemostasis - Glycoproteins • Vasodilator-stimulated phosphoprotein

\begin{abstract}
Background/Aims: Platelets are essential mediators of hemostasis to avoid excessive blood loss. Cirrhosis and chronic liver diseases are characterized by alterations in hemostasis. Alterations in the secondary hemostasis have been well studied, while defects in primary hemostasis, especially the consequences of cholestatic liver disease on platelet function are not well defined. Methods: After bile duct ligation (BDL) platelet activation and thrombus formation were analyzed in mice. Results: BDL in mice had a moderate effect on platelet counts; however, intrinsic platelet activation was strongly reduced upon activation of the collagen receptor GPVI at early time points. 7 days after bile duct ligation, platelets displayed an almost complete loss of activation with reduced agonist-triggered release of alpha and dense granules and expression of integrin $\alpha_{\mathrm{IIb}} \beta_{3}$ on the platelet surface. This activation defects resulted in strongly reduced thrombus formation under flow, reduced platelet adhesion to fibrinogen and bleeding complications in BDL mice as measured by tail bleeding experiments. Mechanistically, elevated nitric oxide and prostacyclin levels induced phosphorylation of Vasodilator-stimulated phosphoprotein (VASP), an established inhibitor of platelet activation. Furthermore increased tissue plasminogen activator in plasma of BDL mice led to enhanced plasmin levels that might be responsible for reduced glycoprotein expression of BDL platelets. Besides, high amounts of bile acids contribute to defective signal transduction as shown in platelets from mice fed with a cholic acid diet. Conclusions: Cholestatic liver disease induces multiple platelet activation defects and impairs thrombus formation responsible for bleeding complications at least in mice.




\section{Introduction}

Platelets are the major players in hemostasis. Upon vessel injury they become activated to form a hemostatic plug important to avoid excessive blood loss. However, uncontrolled platelet activation and aggregation can induce acute thrombotic complications leading to vessel occlusion at sides of atherosclerotic plaque rupture, the most significant pathophysiological mechanism leading to myocardial infarction or ischemic stroke $[1,2]$.

Platelets originate from megakaryocytes in the bone marrow. Upon vessel injury subendothelial matrix components are exposed to blood cells leading to platelet adhesion and aggregation. The initial contact is mediated by platelet glycoprotein (GP) Ib to von Willebrand factor (vWF) bound to exposed collagen. This step allows binding of platelet collagen receptor GPVI to exposed collagen leading to platelet activation with subsequent $\mathrm{Ca}^{2+}$ mobilization, integrin $\alpha_{\mathrm{IIb}} \beta_{3}$ activation, cytoskeletal reorganization and phosphatidylserine exposure [1,2]. Platelet activation includes degranulation of $\alpha-$ and dense granules to release the second wave mediators ADP and thromboxane important to propagate platelet activation [3]. Activated integrin $\alpha_{\mathrm{IIb}} \beta_{3}$ mediates firm adhesion via binding to $\mathrm{vWF}$ on exposed collagen and binds fibrinogen that leads to platelet aggregation and thrombus formation [4]. Furthermore thrombin is released from activated platelets to further activate platelets and to cleave fibrinogen to fibrin to stabilize the hemostatic plug [3].

The hemostatic system is in a susceptible balance between pro- and anti-hemostatic processes to avoid on the one hand bleeding and on the other hand thrombotic complications. Liver failure and cirrhosis are characterized by alterations in secondary hemostasis and hemostatic disturbances. Many proteins involved in hemostasis are produced in the liver and a diseased liver has a reduced capacity to clear activated hemostatic proteins. Moreover, thrombocytopenia and platelet function defects as well as hypercoagulability may play a prominent role in liver disease [5, 6].

While alterations in secondary hemostasis have been well studied, primary hemostasis has received less attention [5, 7]. Thrombocytopenia has been described in patients suffering from acute and chronic liver failure $[8,9]$. Different studies in the past suggested that decreased platelet production is a consequence of low levels of thrombopoietin (tpo) in these patients. Moreover, a reduced half-life of platelets has been shown in some patients as well as the presence of chronic (low-grade) disseminated intravascular coagulation (DIC) [10-12].

Beside low platelet counts upon liver failure, platelet function defects have been described. Platelet aggregation after stimulation with ADP, collagen and arachidonic acid and platelet adhesion to extracellular matrix proteins under flow using denuded segments of rabbit aorta was shown to be reduced. In patients with cirrhosis of the liver, inositol lipid and arachidonic acid metabolism in response to agonists was impaired [13]. Defective platelet-vessel wall interaction has also been described in patients with cirrhosis [14]. Impaired platelet activation and adhesion might be due to defective signal transduction in platelets from cirrhotic patients, an acquired storage pool deficiency [15] and decreased concentration of arachidonic acid that is important for thromboxane A2 production [16]. Furthermore, increased levels of cyclic nucleotides [17] and nitric oxide (NO) [18] might account for impaired platelet function in patients with liver failure.

However, more recent data provide evidence that abnormal hemostasis in patients with liver disease is not indicative for bleeding problems [19]). Pre-operative correction of hemostatic abnormalities in patients with liver transplantation surgery provides evidence that this correction does not reduce but may in fact increase bleeding tendencies $[20,21]$. Routine hemostasis tests fail to reflect hemostatic balance and are sometimes misinterpreted. Thus, additional studies are needed to define diagnostic and treatment strategies to prevent or treat bleeding in patients with liver disease [19].

Although platelet numbers are decreased and various activation defects have been described in patients with chronic liver disease and cirrhosis, the consequences of cholestatic liver disease on platelet activation and aggregation is not well understood. Few 
studies suggest a hypercoagulable state in patients with cholestatic liver disease [22, 23]. Atucha and colleagues described increased $\mathrm{Ca}^{2+}$ responses of platelets from bile duct ligated rats [24]. In contrast to these studies showing platelet hyperactivity, hypoaggregability has been proposed from others with reduced expression of P-selectin, defective aggregation and prolonged bleeding times $[15,18,23,25]$. Effects of bile acids on platelet activation have also been suggested [26]. In the last decades, several experimental rodent models of acute and chronic hepatic failure have been established, for example the experimental obstruction of the extrahepatic biliary system known to initiate a complex cascade of pathological events leading to cholestasis and inflammation [27]. A most commonly used model is the surgical ligation of the common bile duct (bile duct ligation, BDL) to induce obstructive cholestatic injury in rodents instrumental for the analysis of molecular and cellular events that underlie the pathophysiological mechanisms induced by inappropriate bile flow [27]. Here we used this model of complete obstruction of the common bile duct and analyzed altered platelet function and the underlying mechanisms in the acute and sub-acute phase after injury to study the consequences of cholestatic liver disease on hemostasis.

\section{Materials and Methods}

\section{Chemicals and antibodies}

Platelets were activated by ADP (Sigma-Aldrich), Fluorophore-labeled antibodies anti-P-selectinFITC (Wug.E9-FITC, Rat IgG2b, Emfret Analytics) and anti-integrin $\alpha_{\mathrm{IIb}} \beta_{3}$-PE (JON/A-PE, Rat IgG1, Emfret Analytics) were used for flow cytometric analysis.

Thrombin (Roche), CRP (Richard Farndale, University of Cambridge, United Kingdom), PAR-4 activating peptide (AYPGKF, JPT Peptide Technologies), U46619 (Enzo Life Sciences), Collagen Reagent HORM $^{\circledast}$ (Nycomed), human fibrinogen (Sigma-Aldrich), heparin (Ratiopharm), prostacyclin (Calbiochem), apyrase (Sigma-Aldrich), Complete protease inhibitor cocktail (Roche) were purchased.

\section{Animals, diet and Bile Duct Ligation (BDL)}

Specific pathogen-free C57BL/6J mice were obtained from Charles River (Sulzfeld, Germany). All animal experiments were conducted according to the Declaration of Helsinki and the guidelines from Directive 2010/63/EU of the European Parliament on the protection of animals and were approved by local authorities. Where indicated mice were fed ad libitum with either standard rodent chow or rodent chow enriched with $0.5 \%$ cholic acid for seven days (Sniff, Soest, Germany).

Bile duct ligation (BDL) was performed as described elsewhere [28]. Briefly, animals were anesthetized by isoflurane and shaved after intubation and ventilation. A midline incision in the upper abdomen was made to identify, isolate and ligate the common bile duct and the gallbladder with a 6-0 coated vicryl polyglactin fiber from Ethicon (Johnson \& Johnson Medical GmbH, Norderstedt, Germany). The fascia and skin of the abdominal incision were closed with 5-0 vicryl Polyglactin fiber. Sham treatment was performed similarly but without ligation of the bile duct and gallbladder. Animals were monitored until recovery and treated with carprofen $(5 \mathrm{mg} / \mathrm{kg}$ b.w.) after the procedure. After the respective time points animals were anesthetized with i.p. injection of ketamine/sodium pentobarbital solution and serum, liver and spleen were collected.

\section{Platelet preparation}

Platelets were prepared as previously described. $[29,30]$ Briefly, murine blood from retro-orbital plexus was collected and centrifuged at $1800 \mathrm{rpm}(250 \mathrm{~g})$ for 5 minutes at room temperature. To obtain platelet-rich plasma (PRP), the supernatant was centrifuged at $800 \mathrm{rpm}(50 \mathrm{~g})$ for $6 \mathrm{~min}$. PRP was washed twice at $2800 \mathrm{rpm}(650 \mathrm{~g})$ for $5 \mathrm{~min}$ at room temperature and pellet was resuspended in Tyrode's buffer [136 mM NaCl, $0.4 \mathrm{mM} \mathrm{Na}_{2} \mathrm{HPO}_{4}, 2.7 \mathrm{mM} \mathrm{KCl}, 12 \mathrm{mM} \mathrm{NaHCO}_{3}, 0.1 \%$ glucose, $0.35 \%$ bovine serum albumin (BSA), pH 7.4] supplemented with prostacyclin $(0.5 \mu \mathrm{M})$ and apyrase $(0.02 \mathrm{U} / \mathrm{mL})$. Before use, platelets were resuspended in the same buffer and incubated at $37^{\circ} \mathrm{C}$ for $30 \mathrm{~min}$.

\section{KARGER}




\section{Cellular Physiology Cell Physiol Biochem 2017;41:2133-2149

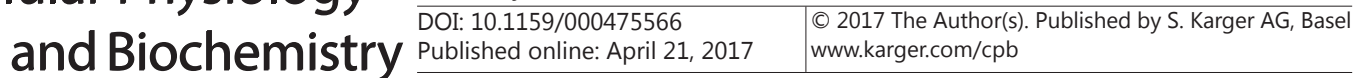

Gowert et al.: Platelets and Cholestatic Liver Disease

\section{Flow cytometry}

Flow cytometric analysis was performed as described elsewhere [31]. Briefly, two-colour analysis of murine platelet activation was performed using fluorophore-labeled antibodies for P-selectin expression (Wug.E9-FITC) and the active form of $\alpha_{\mathrm{IIb}} \beta_{3}$ integrin (JON/A-PE). Heparinized blood was diluted in Tyrode's buffer and washed twice. Blood samples were mixed with antibodies after addition of $1 \mathrm{mM} \mathrm{CaCl}_{2}$ and stimulated with indicated agonists for $15 \mathrm{~min}$ at room temperature. Reaction was stopped by the addition of PBS and samples were analyzed on a FACSCalibur flow cytometer (BD Biosciences).

For analysis of glycoprotein surface expression blood samples were mixed with antibodies and incubated for $30 \mathrm{~min}$ at room temperature. Up-regulation of integrin expression was detected with antibody against integrin $\beta 3$ chain (GPIIIa, CD61, Luc.H11, Emfret analytics).

\section{Serotonin release}

7 days after bile duct ligation, washed platelets from BDL mice and sham controls at a concentration of $0.4 \times 10^{6} / \mu \mathrm{L}$ were activated with the indicated agonists for 2 minutes at $37^{\circ} \mathrm{C}$ and $400 \mathrm{rpm}$ and immediately centrifuged. The level of serotonin released by platelets after activation with CRP and thrombin, respectively, was quantified using a Serotonin ELISA Kit (Enzo Life Sciences AG) according to the manufacturers' protocol.

\section{Platelet adhesion under flow using the flow chamber}

Cover slips ( 24 x $60 \mathrm{~mm}$ ) were coated with $200 \mu \mathrm{g} / \mathrm{mL}$ fibrillar type I collagen (Nycomed) and blocked with $1 \%$ BSA. Tyrode`s buffer was prewarmed at $37^{\circ} \mathrm{C}$. Mice were anesthetized with isoflurane and blood was taken from the retro-orbital plexus and collected in a tube containing $300 \mu \mathrm{L}$ Heparin (20 U/ml in TBS). Whole blood was filled in a $1 \mathrm{ml}$ syringe and perfused over a collagen-coated surface at shear rate of 1000 $\mathrm{sec}^{-1}$ and platelet adhesion and aggregate formation was evaluated from 6 different microscopic areas (x 20, Carl Zeiss) [30, 31].

\section{Platelet adhesion}

Cover slips were coated with $200 \mu \mathrm{g} / \mathrm{mL}$ human fibrinogen and $250 \mu \mathrm{g} / \mathrm{mL}$ fibrillar type I collagen (Nycomed) at $4^{\circ} \mathrm{C}$ overnight and then blocked with 1\% BSA. Washed platelets were resuspended at a concentration of $10^{5}$ platelets/ $\mu \mathrm{L}$ in Tyrode's buffer supplemented with $1 \mathrm{mM} \mathrm{CaCl}_{2}$. Platelets were seeded on cover slips and incubated at room temperature for $60 \mathrm{~min}$ [31].

\section{Quantification of tissue Plasminogen activator (ELISA)}

Tissue Plasminogen activator (tPA) in plasma of BDL mice and sham controls was quantified 7 days after bile duct ligation in mice following the manufacturer's protocol (Uscn Life Science Inc., Tx, USA).

Quantification of prostacycline (PGI2) by ELISA

Prostacycline (PGI2) in plasma of BDL mice and sham controls was determined 7 days after bile duct ligation following the manufacturer's protocol (CUSABIO BIOTECH Co., LTD.).

\section{Quantification of plasmin-antiplasmin complex (PAP) by ELISA}

To determine the level of plasmin 7 in plasma of BDL mice and sham controls, plasmin-antiplasmin complex (PAP) was quantified 7 days after bile duct ligation following the manufacturer's protocol (CUSABIO BIOTECH Co., LTD.).

Quantification of total Nitric Oxide (NO) and Nitrate/Nitrite by ELISA

NO in plasma of BDL mice and sham controls 7 days after bile duct ligation was measured by quantification of the stable NO metabolites nitrate and nitrite following the manufacturer's protocol (R\&D Systems Europe, Ltd., UK).

\section{Western Blot analysis}

Vasodilator-stimulated phosphoprotein (VASP) phosphorylation was determined in platelets that had been incubated with the platelet guanylyl cyclase activator sodium nitroprusside (SNP), the platelet adenylyl cyclase activator prostaglandin $\mathrm{I}_{2}$ (prostacyclin, $\mathrm{PGI}_{2}$ ), phorbol 12-myristate 13-acetate (PMA) or in vehicle alone (rest). Platelets were sedimented by centrifugation and lysates were prepared, separated 
on SDS-polyacrylamide gel and transferred onto polyvinylidene fluoride (PVDF) membrane. Subsequently, the membrane was blocked using $5 \%$ powdered skim milk in PBST (PBS with $0.1 \%$ Tween 20) and probed with appropriate antibody. VASP phosphorylation was detected with phosphorylation specific antibodies (phospho-VASP (Ser157, \#3111) and phospho-VASP (Ser239, \#3114)); total VASP was detected with polyclonal antibody (\#3112, Cell Signaling Tech.).

Phosphorylation of PLC $\gamma 2$ was determined with phosphorylation specific antibodies (phospho-PLC $\gamma 2$ (Tyr759, \#3874); total PLC $\gamma 2$ was detected with polyclonal antibody (\#3872, Cell Signaling Tech.). Western blot analysis was done as described above.

\section{Histology}

For the analysis of tissue injury in liver and megakaryocytes in spleen of bile duct ligated mice, fivemicron sections of paraffin-embedded samples were stained with hematoxylin and eosin.

For immunofluorescence staining of platelets paraffin-embedded liver sections of BDL and sham operated mice were stained with an anti-GPIb $\alpha$ monoclonal antibody (rat anti mouse GPIb $\alpha$ (CD42b), Emfret Analytics, \# M042-0, 1:100) followed by an Alexa-Fluor 568 labeled secondary antibody (goat anti rat, invitrogen, 1:250). Nuclei were identified using DNA staining with 4,6 diamidino-2-phenylindole dihydrochloride (DAPI, Roche, 1:3000). Green auto-fluorescence of erythrocytes was detected with an excitation maximum of $470 \mathrm{~nm}$.

\section{Bleeding time}

Mice were anaesthetized with i.p. injection of ketamine/sodium pentobarbital solution and the tail was transacted $3 \mathrm{~mm}$ from the tip with a scalpel. The tail was immersed in normal saline $\left(37^{\circ} \mathrm{C}\right)$. The time from the incision to the cessation of bleeding was recorded (no blood flow for $1 \mathrm{~min}$ ) as described previously [30].

\section{Statistical analysis}

Data are provided as arithmetic means \pm SEM, statistical analysis was made by one-way ANOVA or student's paired t-test, where applicable.

\section{Results}

BDL causes megakaryopoiesis and temporary mild thrombocytopenia in mice

All bile duct ligated mice showed typical features of cholestasis such as jaundice, enlarged liver and spleen as well as abnormal liver function tests compared to sham operated controls (Fig. 1A-B, Table 1, Table 2). As liver disease is often associated with thrombocytopenia or

Table 1. Liver and spleen weight of mice after 7 days ( $n=3-8$ per group) and mean platelet counts at different time points after BDL or sham surgery are shown ( $\mathrm{n}=5-7$ per group). ${ }^{*} \mathrm{p}<$ $0.05,{ }^{* *} \mathrm{p}<0.01$, sham vs. BDL

\begin{tabular}{lcc}
\hline & \multicolumn{1}{l}{ Sham } & \multicolumn{1}{l}{ BDL } \\
\hline Liver weight $(\mathrm{g})$ & $0.9653 \pm 0.0347$ & $1.5207 \pm 0.1174^{* *}$ \\
Spleen weight $(\mathrm{mg})$ & $71.9 \pm 5.08$ & $96.4 \pm 6.4143^{*}$ \\
Platelet count $\left(\mathrm{x} 10^{5} / \mu \mathrm{L}\right)$ after: & & \\
$24 \mathrm{~h}$ & $9.9 \pm 0.4936$ & $7.26 \pm 0.9726^{*}$ \\
$3 \mathrm{~d}$ & $9.3255 \pm 0.6744$ & $6.2738 \pm 0.5018^{* *}$ \\
$7 \mathrm{~d}$ & $8.3873 \pm 0.3853$ & $9.6843 \pm 1.4332$ \\
\hline
\end{tabular}

Table 2. Serum analysis of animals 7 days after BDL. Liver enzymes (AST, aspartate aminotransferase; ALT, alanine aminotransferase) and alkaline phosphatase (ALP) and bilirubin were measured by LCMS. ${ }^{*} \mathrm{p}<0.05,{ }^{* *} \mathrm{p}<0.01,{ }^{* * *} \mathrm{p}<0.001$, sham vs. BDL

\begin{tabular}{lcc}
\hline & Sham & BDL \\
\hline AST (IU/l) & $23 \pm 10.2$ & $360.3 \pm 52^{* *}$ \\
ALT (IU/l) & $20.3 \pm 10.3$ & $299 \pm 50.9^{* *}$ \\
Bilirubin (mg/dl) & $0.2 \pm 0.0$ & $12.6 \pm 1.2^{* * *}$ \\
ALP (IU/l) & $80.8 \pm 8.9$ & $424.7 \pm 122.9^{*}$ \\
\hline
\end{tabular}




\section{Cellular Physiology and Biochemistry

Fig. 1. Mice showed clear cholestasis, increased megakaryopoiesis in spleen and mild thrombocytopenia at early time points after bile duct ligation (BDL). Mice with BDL were compared to sham controls. (A) Liver histology showed tissue injury and prominent bile infarcts 7 days after BDL ( $n=3-8)$. (B) Spleen sections of BDL mice revealed megakaryopoiesis 7 days after BDL. Scale bar $100 \mu \mathrm{m}(\mathrm{n}=3-8)$. Arrows indicate megakaryocytes in the spleen. (C) Accumulation of platelets in the liver of sham control and BDL mice at indicated time points. Platelets were stained with an anti-GPIb-antibody (CD42b/ GP1b $\alpha$, Emfret, red), RBCs appear green because of their auto-fluorescence. Scale bar $50 \mu \mathrm{m}$.

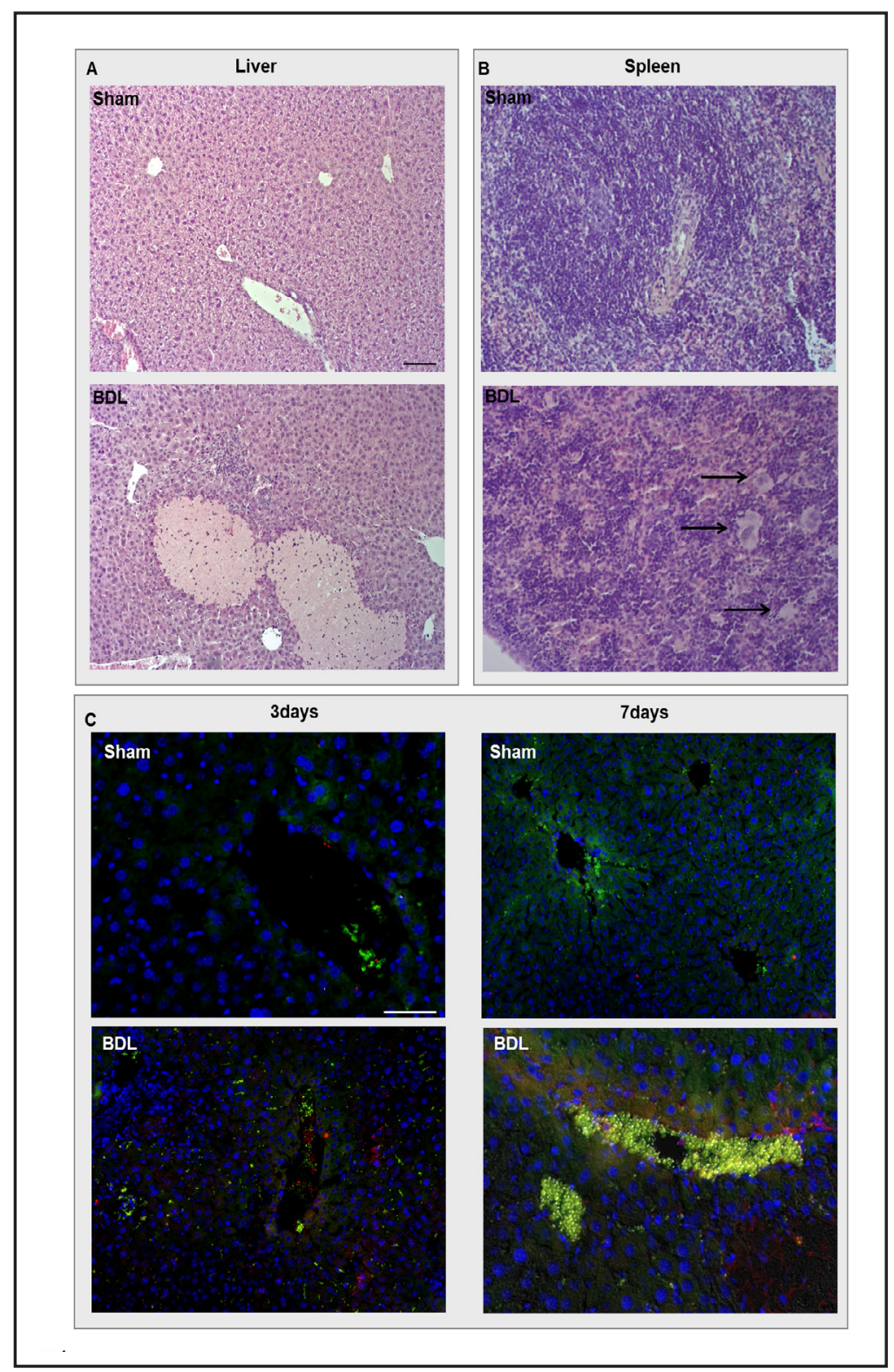

low platelet counts we analyzed the influence of cholestasis on platelet numbers at different time points (Table 1). As expected, platelet counts decreased $24 \mathrm{~h}$ and 3 days after bile duct ligation but increased to normal levels at day 7 . This might be due to megakaryopoiesis in spleen of BDL mice while no megakaryocytes were detected in spleen of sham controls (Fig. 1B). The analysis of white blood cells (WBCs) and erythrocytes revealed increased WBCs 7 days post BDL. The number of red blood cells was not altered $24 \mathrm{~h}$ and 3 days post BDL, but slightly reduced 7 days after bile duct ligation without reaching statistical significance (for all online suppl. material, see www.karger.com/doi/10.1159/000475566, Fig. S1). 3 and 7 days post BDL platelets accumulate in vessels of the liver and in the sinusoidal space while almost no platelets were detected in the liver of sham control mice (Fig. 1C).

Platelets show activation defects in response to stimulation of the collagen receptor GPVI 3 days after $B D L$

To analyze platelet activation, degranulation of $\alpha$-granules (P-selectin expression) and integrin $\alpha_{\mathrm{IIb}} \beta_{3}$ activation (JON/A-binding) in response to different agonist concentrations were measured in washed whole blood using flow cytometry. Platelets without agonist confirmed 


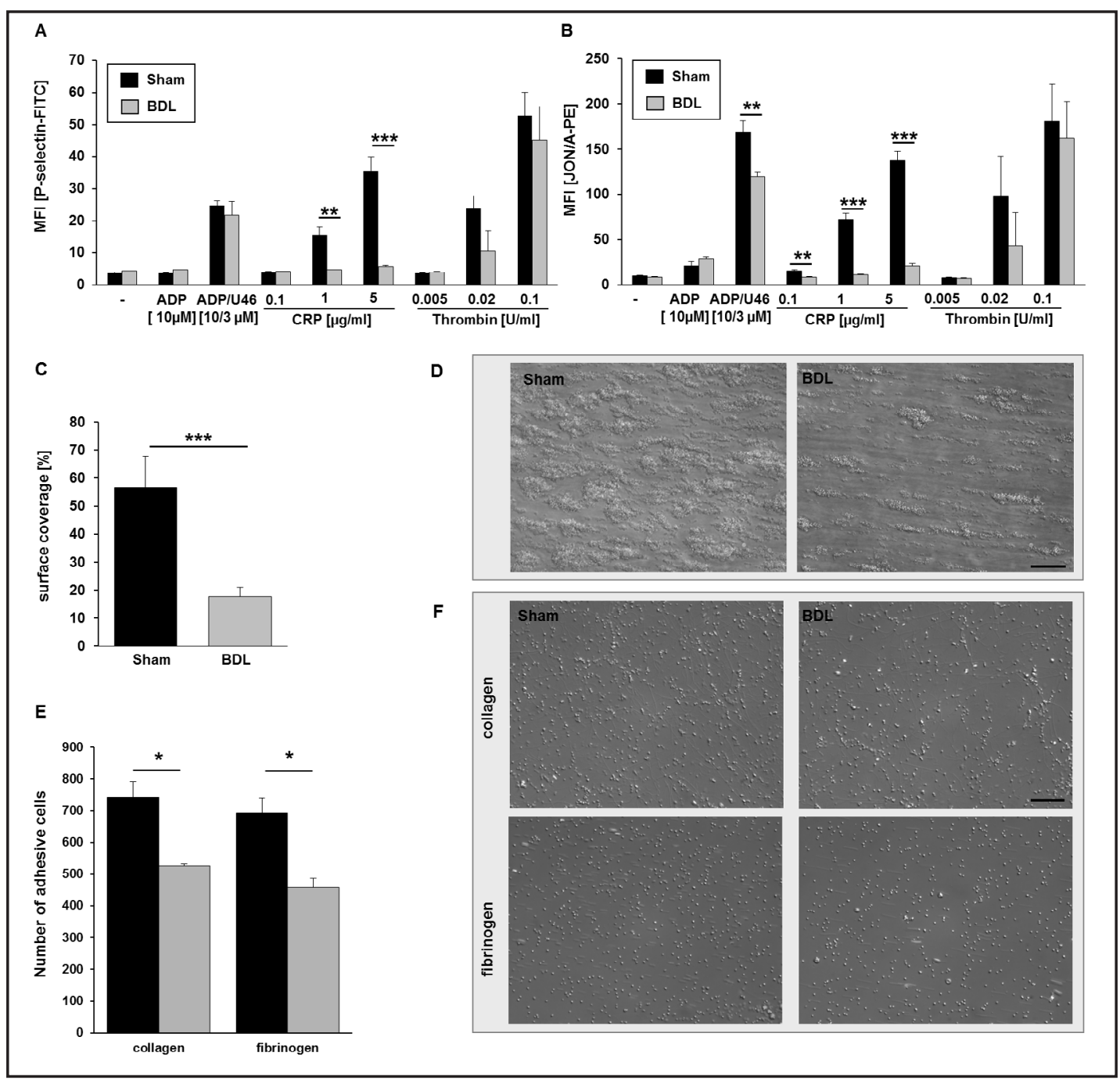

Fig. 2. Cholestasis affects intrinsic platelet activation 3 days after BDL. Washed blood was incubated with indicated agonists for $15 \mathrm{~min}$. in the presence of P-selectin (A) and JON/A (B) antibody that only binds to activated integrin $\alpha_{\mathrm{II}} \beta_{3}$. Platelets were gated by their forward and side scatter characteristics. The mean fluorescence intensity (MFI) is shown for each measurement $(n=5)$. Thrombus formation under flow: Whole blood was perfused over a collagen matrix at a shear rate of $1000 \mathrm{sec}^{-1}$. Statistical analysis (C) and representative images (D) of BDL mice and sham controls $(n=4)$. (E) Defective platelet adhesion on extracellular matrix proteins, (E) Statistical analysis and (F) representative pictures $(n=4)$. Bar graphs depict mean values \pm S.E.M. $* \mathrm{P}<0.05,{ }^{* *} \mathrm{P}<0.01{ }^{* * *} \mathrm{P}<0.001$.

resting state of platelets and served as negative controls. Interestingly, integrin activation in response to collagen-related peptide (CRP) known to stimulate the immunoreceptor tyrosine-based activation motif (ITAM)-coupled receptor glycoprotein (GP)VI was strongly impaired at all concentrations tested. G-protein coupled receptor (GPCR) stimulation with ADP+U46619 (thromboxane A2 (TxA2) analogue) led to decreased integrin activation as well, while P-selectin exposure was not affected when platelets were stimulated with ADP/ U46619 and thrombin, respectively (Fig. 2A-B).

To explore defective platelet activation under more physiological conditions, whole blood of BDL mice and sham controls was perfused over a collagen coated surface under flow to investigate platelet adhesion and thrombus formation ex vivo. Both platelets from BDL mice and sham controls adhered to the collagen matrix and formed aggregates. However, while platelets from sham controls were able to form large three-dimensional thrombi, only 


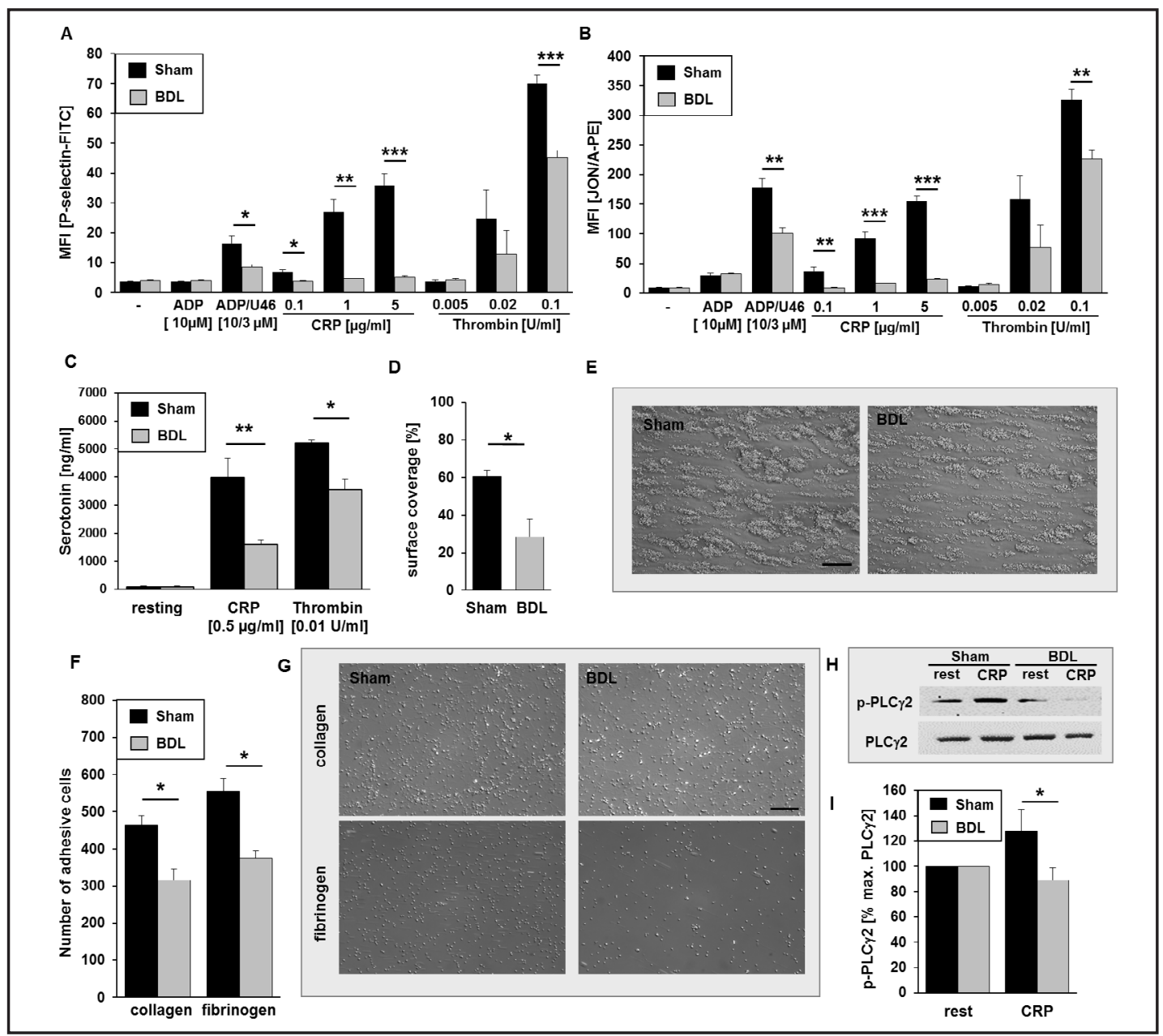

Fig. 3. Extensive defects of intrinsic platelet activation 7 days after BDL. (A-B) Platelet activation was measured by flow cytometry and MFI was detected for P-selectin (A) and activated integrin $\alpha_{\mathrm{IIb}} \beta_{3}(\mathrm{JON} / \mathrm{A})(\mathrm{B})(\mathrm{n}=4-7)$. (C) The release of serotonin as marker for degranulation of dense granules was measured by ELISA following platelet stimulation with CRP and thrombin as indicated ( $\mathrm{n}=3-6)$. (D-E) Flow chamber experiments are shown (shear rate 1000 $\mathrm{sec}^{-1}$ ). Analysis of surface coverage (D) and representative pictures (E) comparing thrombus formation with whole blood of BDL mice and sham controls $(n=4)$. (F) Platelet adhesion to collagen (left panel) and fibrinogen (right panel) is shown. (G) Representative images showing adhesion of platelet from BDL mice and sham controls $(n=4)$. $(H)$ Representative Western blots showing phospho-PLC $\gamma 2$ in resting and CRP stimulated platelets of sham control and BDL mice. (I) Evaluation of PLC $\gamma 2$ phosphorylation as \% of max. PLC $\gamma 2$ expression $(n=4)$. Bar graphs depict mean values \pm S.E.M. $* \mathrm{P}<0.05,{ }^{* *} \mathrm{P}<0.01 * * * \mathrm{P}<0.001$.

small aggregates and small thrombi developed with whole blood from BDL mice (Fig. 2CD). Thus, the surface covered by thrombi was significantly reduced using whole blood from cholestatic mice.

To elucidate the ability of platelets to adhere to different matrix proteins, cell adhesion experiments were performed. Platelets were allowed to adhere to collagen and fibrinogen, respectively and number of firmly adherent cells was analyzed (Fig. 2E-F). After $60 \mathrm{~min}$ significantly reduced cell adhesion on collagen (Fig. 2F, upper panel) and fibrinogen (Fig. 2F, lower panel) was detected with platelets from BDL mice taken 3 days after bile duct ligation.

Taken together, cholestasis in mice induced defective platelet activation and adhesion leading to impaired thrombus formation under flow 3 days after bile duct ligation. 


\section{Cellular Physiology Cell Physiol Biochem 2017;41:2133-2149 and Biochemistry Published online: April 21, $2017 \quad \begin{aligned} & \text { DOI: 10.1159/000475566 } 2017 \text { The Author(s). Published by S. Karger AG, Basel } \\ & \text { www.karger.com/cpb }\end{aligned}$ \\ Gowert et al.: Platelets and Cholestatic Liver Disease}

Strongly reduced platelet activation following stimulation of GPCR and ITAM coupled signaling pathways 7 days post BDL

Next we investigated if platelet activation defects 3 days after BDL are only temporary or if defective thrombus formation persists for a longer time period. Thus, platelet activation and adhesion in vitro and thrombus formation on collagen under flow ex vivo were determined 7 days after induction of cholestasis in mice. In contrast to early platelet activation defects after 3 days, we now found strongly reduced P-selectin exposure and reduced activation of integrin $\alpha_{\mathrm{IIb}} \beta_{3}$ with all agonists tested (Fig. 3A-B). Interestingly, only high concentrations of thrombin led to platelet activation defects while intermediate concentrations showed reduced platelet activation only by trend without reaching statistical significance (Fig. $3 \mathrm{~A}-\mathrm{B})$. Moreover, degranulation of dense granules as measured by serotonin release after stimulation with CRP and thrombin, respectively, was reduced when platelets from BDL mice were analyzed compared to sham controls (Fig. 3C). Again and according to activation defects observed with flow cytometry, we found significantly reduced thrombus formation on collagen under flow (Fig. 3D-E). Furthermore, the analysis of platelet adhesion on collagen and fibrinogen, respectively, was impaired 7 days after BDL (Fig. 3F-G). Another key regulator of platelet activation and calcium mobilization is the phosphorylation of PLC $\gamma 2$. As shown in Fig. 3H-I, PLC $\gamma 2$ phosphorylation in response to CRP was significantly reduced in platelets from BDL mice compared to sham controls indicating that platelet stimulation and calcium mobilization essential for integrin activation and degranulation is impaired upon cholestasis. Taken together, these data suggest that defects in platelet activation persist 7 days after BDL and might continue for a longer time period after induction of cholestasis independent of the platelet count.

Multiple defects are responsible for strongly reduced platelet activation following BDL

To investigate the mechanisms responsible for defective platelet activation we first examined the levels of the stable NO metabolites nitrate and nitrite by ELISA, because it is known that cholestasis induces altered nitric oxide (NO) metabolism [18]. As shown in Fig. 4A, high levels of nitrate and nitrite were detected in plasma of BDL mice 7 days after induction of cholestasis. In contrast, the concentration of prostaglandin $\mathrm{I}_{2}$ (prostacyclin, $\mathrm{PGI}_{2}$ ) was only slightly enhanced in plasma of BDL mice without reaching statistical significance (Fig. 4B). High levels of $\mathrm{NO}$ and $\mathrm{PGI}_{2}$ in plasma induce the phosphorylation of VASP via activation of the adenylyl cyclase (AC) and/or the guanylyl cyclase (GC) to enhance cAMP and cGMP levels followed by the activation of protein kinase A (PKA) and protein kinase G (PKG) and subsequent phosphorylation of VASP at Ser157 and/or Ser 239 [32]. To test if platelet inhibition is due to increased VASP phosphorylation, a potent inhibitor of platelet activation, we stimulated platelets with $\mathrm{PGI}_{2}$ and phorbol 12 -myristate 13-acetate (PMA) to analyze VASP phosphorylation at residue Ser157. We found a slight but significant increase in VASP phosphorylation upon $\mathrm{PGI}_{2}$ treatment using platelets from BDL mice (Fig. 4C-D). Further Western blot experiments showed that VASP is strongly phosphorylated at residue Ser239, when BDL platelets were stimulated with sodium nitroprusside (SNP) (Fig. 4C, $4 \mathrm{E})$ suggesting that enhanced production of prostacyclins and NO upon cholestasis affect platelet activation.

The expression of integrin $\alpha_{\mathrm{IIb}} \beta_{3}$ is highly up-regulated upon platelet stimulation and plays an important role in firm adhesion and platelet aggregate formation [4] To analyze agonist-induced expression of integrin $\alpha_{\mathrm{IIb}} \beta_{3}$ on the platelet membrane 7 days after BDL, mean fluorescence intensity of CD61-FITC was determined by flow cytometry. As shown in Fig. 4F, integrin expression at the platelet surface was significantly reduced with platelets from BDL mice compared to sham controls when platelets have been activated with CRP or thrombin to stimulate the ITAM-coupled and the GPCR signaling pathway in platelets, respectively (Fig. 4F).

We next wanted to know if -beside increased phosphorylation of VASP and decreased up-regulation of integrin expression in BDL platelets- other mechanisms account for reduced platelet activation and thrombus formation after cholestasis in mice. The analysis 
Fig. 4. Multiple defects are responsible for strongly reduced activation of platelets 7 days after BDL. (A) The concentration of the NO metabolites nitrate and nitrite were determined by ELISA $(n=10)$. (B) Total levels of prostacycline in plasma were detected by ELISA ( $\mathrm{n}$ = 5). (C-E) Moderately increased phosphorylation of the platelet inhibitor VASP at Ser157 (C-D) and strongly phosphorylated VASP-Ser239 (C, E) in platelets from $\mathrm{BDL}$ mice $(\mathrm{n}=6)$. (F) Agonist-induced expression of integrin $\alpha_{\mathrm{IIb}} \beta_{3}$ is reduced upon CRP and thrombin activation of platelets from BDL mice compared to controls (Sham) (n = 4-9). (G) Reduced glycoprotein expression on membrane of platelets from BDL mice at day $7(\mathrm{n}=5)$. (H) Enhanced tissue Plasminogen Activator (t-PA) in plasma of BDL mice ( $\mathrm{n}=4$ ). (I) Total levels of plasmin in plasma of BDL mice and sham controls were determined by the analysis of plasmin-antiplasmin complexes and ELISA $(n=5)$. Bar graphs depict mean values \pm S.E.M. $* \mathrm{P}<0.05,{ }^{* *} \mathrm{P}<0.01 * * * \mathrm{P}<0.001$.

of glycoprotein expression on the platelet membrane by flow cytometry revealed reduced expression of GPIb, GPVI, integrin $\alpha 5$ and integrin $\beta 3$ on the membrane of BDL platelets (Fig. $4 G)$. Proteolysis of platelet receptors by plasmin is well known [33, 34]. To examine if high levels of plasmin are responsible for reduced glycoprotein expression of BDL platelets we measured tissue plasminogen activator (tPa) in plasma of BDL mice by ELISA (Fig. 4H). tPa levels were strongly increased in plasma of BDL mice 7 days after induction of cholestasis. To test if increased tPa levels induce an increase in plasmin concentration, we determined plasmin-antiplasmin complexes by ELISA. In line with increased levels of tPa we found significantly enhanced plasmin-antiplasmin complexes in plasma of BDL mice 7 days after induction of cholestasis (Fig. 4I) providing evidence for plasmin induced proteolysis of platelet membrane receptors. However, activation-induced shedding of glycoproteins such as GPIb (see supplementary material, Fig. S2A) and GPVI (Fig. S2B) was unaltered in platelets from BDL mice compared to sham controls.

\section{KARGER}




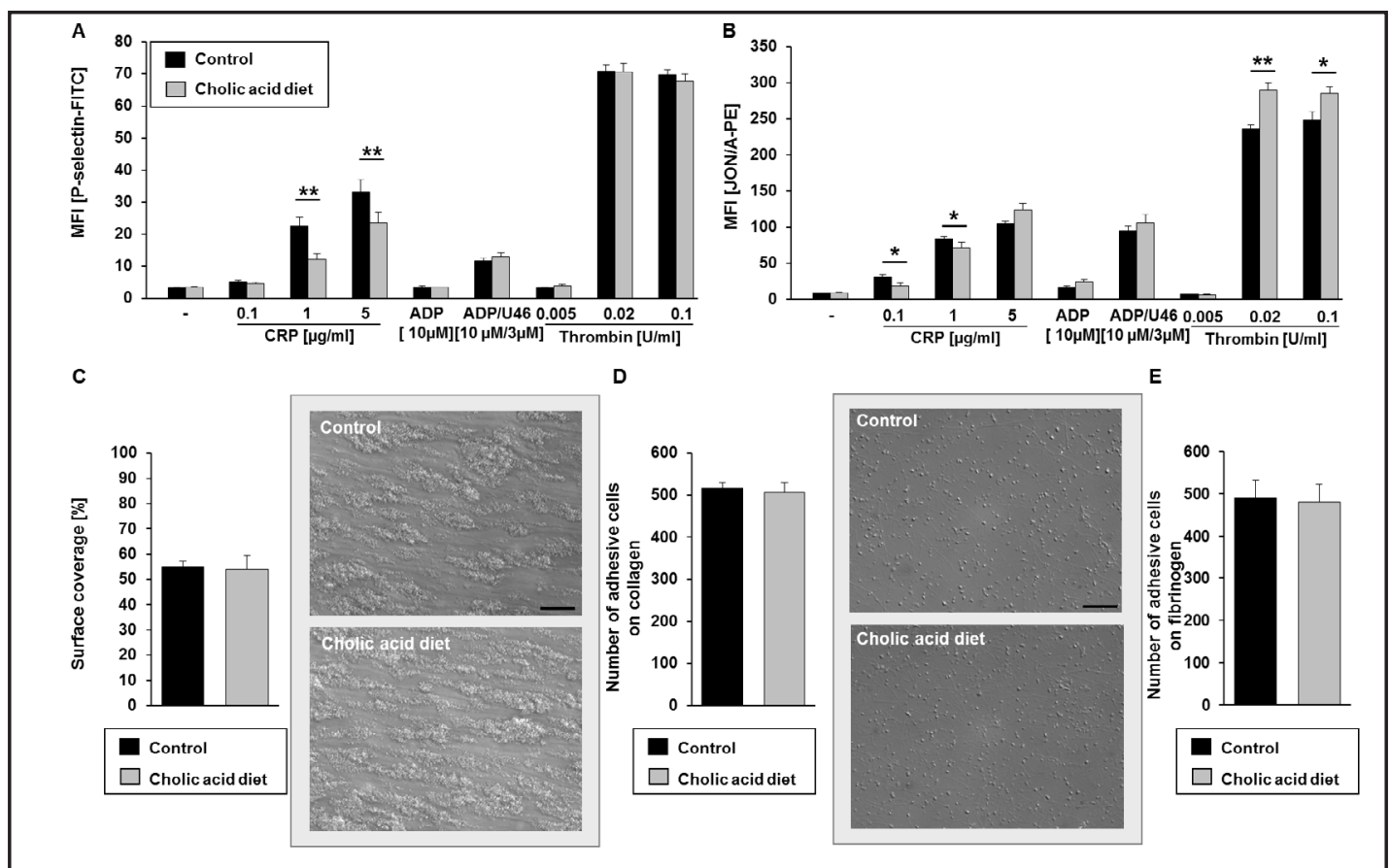

Fig. 5. Bile acids account for platelet activation defects. Mice were fed either with standard rodent chow or rodent chow enriched with $0.5 \%$ cholic acid for seven days. (A, B) Reduced activation of platelets from BDL mice was measured after stimulation with CRP but not with ADP, ADP/U46619 or thrombin $(n=5)$. (C) Analysis of thrombus formation under flow was shown to be not altered. Statistical analysis (upper panel) and representative images (lower panel) $(n=5)$. No defects in static adhesion of platelets on collagen (D, statistical analysis (right panel), representative pictures (left panel)) and fibrinogen (E) were detected. $(\mathrm{n}=5)$. Bar graphs depict mean values \pm S.E.M. $* \mathrm{P}<0.05$, ${ }^{* *} \mathrm{P}<0.01$.

\section{High levels of bile acids account for activation defects}

Bile duct ligation is a well-known animal model for obstructive cholestasis and induces bile stasis, increased biliary pressure, bile infarcts and elevation of liver and serum bile acid levels. Bile acids have been shown to alter platelet activation [35] and aggregation [26] and are elevated in BDL mice and in mice fed with a cholic acid diet [36]. To test, if bile acids account for the observed activation defects of BDL platelets, a chow enriched with $0.5 \%$ cholic acid, a physiological bile acid in the mouse, was given ad libitum for 7 days. A control group was fed with standard rodent chow. No significant changes in platelet count (see supplementary material, Fig. S3A) or numbers of other blood cells were observed after 7 days of cholic acid feeding (see supplementary material, Fig. S3B). Flow cytometric analysis revealed moderately but significantly reduced P-selectin exposure and integrin activation (JON/A binding) following stimulation with CRP (Fig. 5A-B). No alterations in platelet activation were detected in response to ADP and ADP+U46619 (Fig. 5A-B). However, thrombus formation under flow was not altered when whole blood from cholic acid fed mice was used compared to controls (Fig. 5C). Adhesion of platelets to collagen (Fig. 5D) and fibrinogen (Fig. 5E) again showed no differences between both groups.

Taken together, bile acids moderately alter platelet activation without affecting thrombus formation and platelet adhesion. Thus bile acids may account for activation defects in BDL mice.

Prolonged bleeding time in mice 7 days after $B D L$

Bleeding complications have been described in patients suffering from liver disease and cirrhosis $[5,6]$. To investigate the effects of defective platelet activation and thrombus formation on hemostasis, tail bleeding times of BDL mice and sham controls were measured 
Fig. 6. Prolonged bleeding times in mice 7 days after bile duct ligation. To investigate the effects of cholestasis on hemostasis, tail bleeding times of BDL and Sham mice were measured 3 days and 7 days after BDL and compared to C57BL/6 mice without any intervention $(n=4-8)$. Bar graphs depict mean values \pm S.E.M. ${ }^{* * *} \mathrm{P}<0.001$.

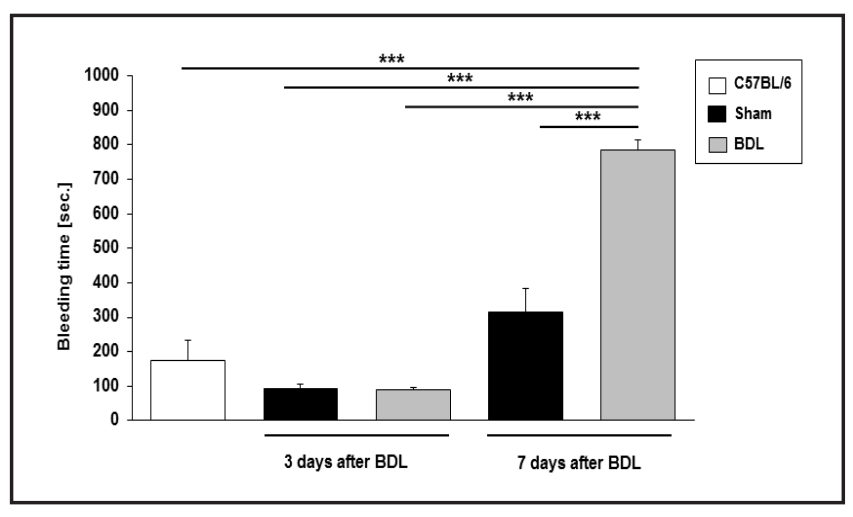

after amputating the tail tip of mice. As shown in Fig. 6, time to arrest bleeding at sites of a defined tail wound was not altered 3 days after bile duct ligation but strongly increased 7 days thereafter $(314.0 \pm 75.3 \mathrm{sec}$ vs. $784.3 \pm 21.0 \mathrm{sec}, \mathrm{p}=0.0005)$. Thus, defective platelet activation and thrombus formation upon cholestatic liver disease impairs hemostasis that might lead to bleeding complications in mice.

Sustained defects of platelet activation in mice 21 days after $B D L$

Impaired platelet activation and thrombus formation was shown 3 days and 7 days after bile duct ligation (Fig. 2-3). To investigate, if activation defects continue over time, we measured JON/A binding 21 days after bile duct ligation. While platelet counts did not differ between BDL mice and sham controls (Fig. 7A), we still detected reduced glycoprotein expression (Fig. 7B) and defective integrin activation using different agonists that stimulate GPCR- and ITAM-coupled receptors (Fig. 7C). This data suggests sustained defects of platelet activation after bile duct ligation in mice.

\section{Discussion}

The present study reveals that cholestatic liver disease lead -beside a transient mild thrombocytopenia- to massive platelet activation defects and critically altered thrombus formation under flow resulting in bleeding complications in BDL mice. According to the present observations multiple effects influence platelet activation and thrombus formation under conditions of cholestasis. Increased production of $\mathrm{PGI}_{2}$ and $\mathrm{NO}$ by endothelial cells induced strong phosphorylation of VASP that together with high bile acid levels is responsible at least in part for defective platelet activation. Furthermore, decreased activation dependent expression of integrin $\alpha_{\mathrm{II}} \beta_{3}$ and reduced glycoprotein expression caused by high levels of tPa and plasmin impact platelet activation and thrombus formation. Taken together, these results indicate that cholestatic liver disease induces multiple platelet activation defects with impaired thrombus formation in vitro and defective hemostasis in vivo.

In the past different studies showed alterations in secondary hemostasis while less attention has been drawn to platelet activation and primary hemostasis, especially after induction of cholestasis [5, 7]. One aspect of altered hemostasis might be due to alterations in platelet counts. Mild to moderate thrombocytopenia has been described in patients with liver disease [5] that might be caused by altered levels of thrombopoietin, increased platelet sequestration in the spleen due to splenomegaly or reduced half-life of platelets [12, 37-39]. Upon bile duct ligation, we only measured moderately reduced platelet counts at early time points while platelet counts reach normal levels at day 7 post BDL suggesting that platelet activation defects are responsible for impaired hemostasis. Thrombocytopenia at early time points might be due to increased sequestration of platelets in the spleen and accumulation of platelets in vessels and in the sinusoidal space of the liver upon cholestasis (Fig. 1C) that is balanced by increased megakaryopoiesis in the spleen (Fig. 1B). 


\section{Cellular Physiology Cell Physiol Biochem 2017;41:2133-2149

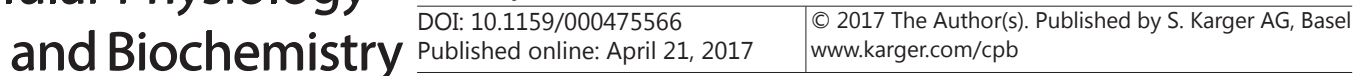 \\ Gowert et al.: Platelets and Cholestatic Liver Disease}

Fig. 7. Sustained defects of platelet activation in mice 21 days after bile duct ligation. (A) Platelet counts 21 days after bile duct ligation is not altered $(n=3)$. (B) Reduced glycoprotein expression on the membrane of platelets 21 days after bile duct ligation is measured by flow cytometry ( $n=3$ ). (C) 21 days after bile duct ligation stimulation of platelets with indicated agonists revealed reduced activation of integrin $\alpha_{\mathrm{IIb}} \beta_{3}$ as measured by JON/A binding ( $\mathrm{n}=$ 3). Bar graphs depict mean values \pm S.E.M. $* \mathrm{P}<0.05$, $* * \mathrm{P}<0.01$.

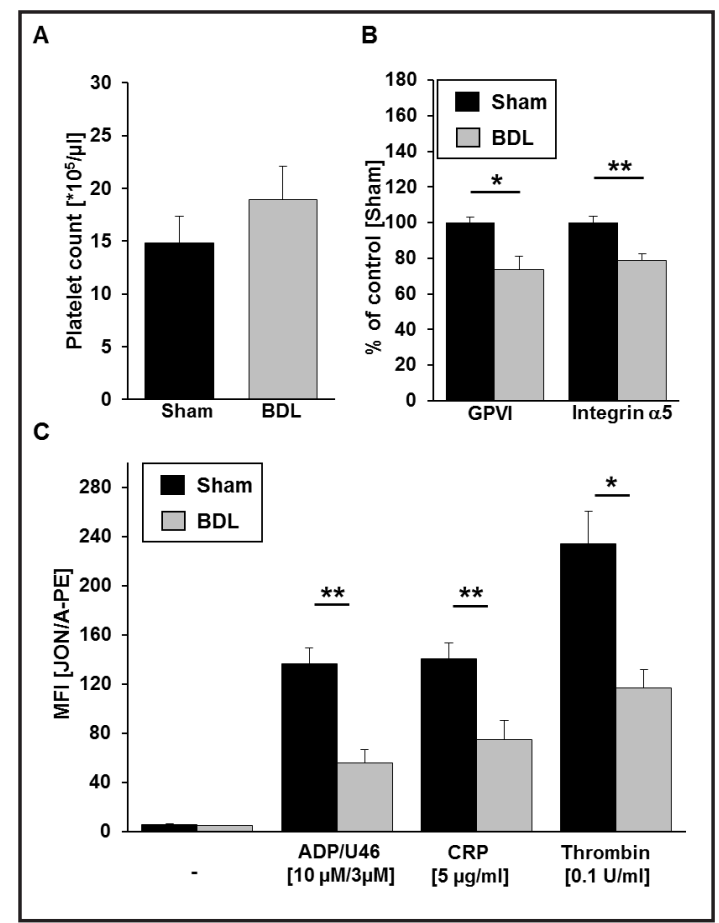

More important, cholestatic liver disease induced alterations in platelet activation leading to platelets that respond only weak to high doses of thrombin and show no responses at all to collagen receptor stimulation. Platelet function defects have been discussed for a long time in patients with chronic or acute liver disease. Platelet aggregation has been shown to be defective as well as platelet-vessel wall interactions [5]. However, signal transduction mechanisms underlying these defects are still missing although few studies tried to correlate increased NO levels upon experimental cirrhosis and impaired thromboxane production with impaired platelet function $[16,18]$.

In the present study we provided strong evidence that different mechanisms affect platelet activation and thrombus formation. Decreased thrombus formation under flow conditions is due to decreased platelet counts (day 3 after BDL) and defective platelet activation. First, high levels of phosphorylated VASP suggest the formation of cAMP and cGMP caused by high $\mathrm{NO}$ and $\mathrm{PGI}_{2}$ levels produced by endothelial cells under cholestatic conditions that strongly inhibit platelet activation [32]. Second, high tPA levels were described in patients with liver failure that may result from either enhanced secretion from ECs or diminished clearance by the diseased liver [40]. Different in vitro studies have shown that high plasmin levels lead to plasmin-mediated cleavage of platelet surface receptors such as GPIb [33] and $\alpha_{\text {II }} \beta_{3}$ [34]. Thus it is tempting to speculate that proteolysis takes place upon high tPA levels in plasma of BDL mice. This is further supported by high plasmin levels in plasma of BDL mice providing first evidence for reduced glycoprotein expression of platelet GPIb, GPVI, integrin $\alpha 5$ and integrin $\beta 3$ upon cholestasis in vivo. Interestingly, inactivation of tPA accelerates liver injury after BDL [41]. This data was supported by other studies demonstrating a critical role for plasminogen activator inhibitor-1 (PAl-1) in cholestatic liver injury $[42,43]$. PAl-1 deficiency protects bile duct ligated mice and correlates with increased tPA and reduces liver fibrosis through activation of tPA.

Third, high levels of bile acids might account for defective platelet activation although bile acids alone showed only moderate effects on platelet activation that did not result in impaired thrombus formation under flow. However, bile acid levels are higher in serum of BDL mice (Sham $5.7 \mu \mathrm{mol}$ vs. $1.595 \mu \mathrm{mol} / \mathrm{l} 3$ days after BDL and $1.252 \mu \mathrm{mol} / \mathrm{l} 7$ days after BDL) compared to cholic acid fed mice (329 $\mu \mathrm{mol} / \mathrm{l})$ that might be responsible for the mild activation defects observed in mice with cholic acid diet [36]. In line with these 
results previous studies have demonstrated moderately but significantly reduced platelet aggregation after bile duct ligation in rats [26].

Few studies in the past analyzed platelet activation after bile duct ligation in rodents. Altered $\mathrm{Ca}^{2+}$ signaling was described in bile duct ligated rats [24]. Atucha and colleagues found increased $\mathrm{Ca}^{2+}$ mobilization following thrombin stimulation. In contrast, defective calcium entry and mobilization after bile duct ligation in rats was shown before [44-46]. Calcium signaling is an important key mechanism in platelet function essential for integrin activation, degranulation and cytoskeletal reorganization of platelets [47]. Different mechanisms modulate calcium signaling in platelets such as upregulation of the calcium channel Orai1 at the platelet membrane after platelet stimulation [48] and modulation of cytosolic calcium activity by the calcium activated Cl-channel Ano6 [49]. Besides, calcium signaling in platelets is associated with peripheral artery disease in type2 diabetes [50].

Inhibited platelet activation was found by Witters and colleagues who analyzed bile duct ligated rats as well [51]. The authors provided evidence for ADP-degrading enzymes that inhibit platelet activation upon cholestasis in rats. In contrast to the results presented here they found normal intrinsic platelet activation. In this study flow cytometric analysis of platelet activation was performed in washed blood. We found defective platelet activation under these conditions suggesting that intrinsic platelet activation is responsible for defective thrombus formation and bleeding complications in mice. These results were supported by high levels of phospho-VASP that might be responsible for defective intrinsic platelet activation upon cholestasis in mice.

Another key regulator of cholestasis is sphingosine 1 phosphate (SIP) that regulates pathological changes during cholestasis [52]. S1P mediates the homing of bone marrow cells and thus plays an important role in cholestasis induced liver fibrosis. Moreover, sphingosine 1 phosphate [53] signaling is essential in the clotting system and is released by platelets. Thus, S1P might play an important role in cholestasis-induced alterations of platelets. However, further studies in future are needed to analyze if S1P in platelets plays a role in platelet activation defects and impaired hemostasis upon cholestatic liver disease.

As shown in Table 2, cholestatic liver injury is accompanied by increased levels of bilirubin. Bilirubin was shown to trigger anemia 21 days after BDL in mice by inducing erythrocyte death [28]. Here we also found decreasing numbers of erythrocytes 7 days post BDL by trend (not significant). Furthermore, bilirubin is discussed as anti-thrombotic agent as patients suffering from Gilbert's syndrome (GS) display hyperbilirubinemia with remarkable reduced risk of mortality from cardiovascular disease [54]. Bilirubin inhibits platelet activation and potentially organ infarction by an increased antioxidant status that might also play a role in cholestatic liver disease. Thus, bilirubin might be another mechanism for reduced platelet activation upon BDL in mice. Further studies are needed to gain insight in bilirubin mediated effects on platelet activation and thrombus formation, especially under cholestatic conditions.

In conclusion, we demonstrate that high $\mathrm{NO}, \mathrm{PGI}_{2}$ and $\mathrm{tPA} /$ plasmin plasma levels derived from altered endothelial cell signaling in cholestatic mice and increased bile acids induce platelet activation defects. These defects translated into reduced thrombus formation under flow ex vivo and defective hemostasis in BDL mice. Further studies in patients are needed to explore if cholestatic liver disease induces alterations in hemostasis with bleeding complications or hypercoagulability.

\section{Acknowledgements}

We thank Martina Spelleken for providing outstanding technical assistance.

This study was supported by grant from the Deutsche Forschungsgemeinschaft, Sonderforschungsbereich 974, Düsseldorf. 


\section{Cellular Physiology Cell Physiol Biochem 2017;41:2133-2149 \begin{tabular}{l|l} 
DOI: 10.1159/000475566 & Ond Biochemistry \\
Published online: April 21, 2017 & $\begin{array}{l}\text { 2017 The Author(s). Published by S. Karger AG, Basel } \\
\text { www.karger.com/cpb }\end{array}$
\end{tabular}}

Gowert et al.: Platelets and Cholestatic Liver Disease

\section{Disclosure Statement}

The authors of the manuscript declare that they have no conflict of interest to declare.

\section{References}

1 Ruggeri ZM, Mendolicchio GL: Adhesion mechanisms in platelet function. Circ Res 2007;100:1673-1685.

2 Savage B, Almus-Jacobs F, Ruggeri ZM: Specific synergy of multiple substrate-receptor interactions in platelet thrombus formation under flow. Cell 1998;94:657-666.

3 Ruggeri ZM: Platelets in atherothrombosis. Nat Med 2002;8:1227-1234.

4 Nieswandt B, Varga-Szabo D, Elvers M: Integrins in platelet activation. J Thromb Haemost 2009;7:206-209.

5 Lisman T, Leebeek FW, de Groot PG: Haemostatic abnormalities in patients with liver disease. J Hepatol 2002;37:280-287.

6 Lisman T, Porte RJ: Activation and regulation of hemostasis in acute liver failure and acute pancreatitis. Semin Thromb Hemost 2010;36:437-443.

-7 Caldwell SH, Hoffman M, Lisman T, Macik BG, Northup PG, Reddy KR, Tripodi A, Sanyal AJ: Coagulation disorders and hemostasis in liver disease: Pathophysiology and critical assessment of current management. Hepatology 2006;44:1039-1046.

8 Gallus AS, Lucas CR, Hirsh J: Coagulation studies in patients with acute infectious hepatitis. Br J Haematol 1972;22:761-771.

9 Kelly DA, Tuddenham EG: Haemostatic problems in liver disease. Gut 1986;27:339-349.

10 Ben-Ari Z, Osman E, Hutton RA, Burroughs AK: Disseminated intravascular coagulation in liver cirrhosis: Fact or fiction? Am J Gastroenterol 1999;94:2977-2982.

11 Carr JM: Disseminated intravascular coagulation in cirrhosis. Hepatology 1989;10:103-110.

12 Stein SF, Harker LA: Kinetic and functional studies of platelets, fibrinogen, and plasminogen in patients with hepatic cirrhosis. J Lab Clin Med 1982;99:217-230.

13 Laffi G, Cominelli F, Ruggiero M, Fedi S, Chiarugi VP, La Villa G, Pinzani M, Gentilini P: Altered platelet function in cirrhosis of the liver: Impairment of inositol lipid and arachidonic acid metabolism in response to agonists. Hepatology (Baltimore, Md) 1988;8:1620-1626.

14 Ordinas A, Escolar G, Cirera I, Vinas M, Cobo F, Bosch J, Teres J, Rodes J: Existence of a platelet-adhesion defect in patients with cirrhosis independent of hematocrit: Studies under flow conditions. Hepatology (Baltimore, Md) 1996;24:1137-1142.

15 Laffi G, Marra F, Gresele P, Romagnoli P, Palermo A, Bartolini O, Simoni A, Orlandi L, Selli ML, Nenci GG, et al.: Evidence for a storage pool defect in platelets from cirrhotic patients with defective aggregation. Gastroenterology 1992;103:641-646.

-16 Owen JS, Hutton RA, Day RC, Bruckdorfer KR, McIntyre N: Platelet lipid composition and platelet aggregation in human liver disease. J Lipid Res 1981;22:423-430.

17 Laffi G, Marra F, Failli P, Ruggiero M, Cecchi E, Carloni V, Giotti A, Gentilini P: Defective signal transduction in platelets from cirrhotics is associated with increased cyclic nucleotides. Gastroenterology 1993;105:148156.

18 Albornoz L, Bandi JC, Otaso JC, Laudanno 0, Mastai R: Prolonged bleeding time in experimental cirrhosis: Role of nitric oxide. J Hepatol 1999;30:456-460.

19 Lisman T, Porte RJ: Rebalanced hemostasis in patients with liver disease: Evidence and clinical consequences. Blood 2010;116:878-885.

20 de Boer MT, Christensen MC, Asmussen M, van der Hilst CS, Hendriks HG, Slooff MJ, Porte RJ: The impact of intraoperative transfusion of platelets and red blood cells on survival after liver transplantation. Anesth Analg 2008;106:32-44, table of contents.

-21 Massicotte L, Capitanio U, Beaulieu D, Roy JD, Roy A, Karakiewicz PI: Independent validation of a model predicting the need for packed red blood cell transfusion at liver transplantation. Transplantation 2009;88:386-391.

22 Biagini MR, Tozzi A, Marcucci R, Paniccia R, Fedi S, Milani S, Galli A, Ceni E, Capanni M, Manta R, Abbate R, Surrenti C: Hyperhomocysteinemia and hypercoagulability in primary biliary cirrhosis. World J Gastroenterol 2006;12:1607-1612. 


\section{Cellular Physiology Cell Physiol Biochem 2017;41:2133-2149

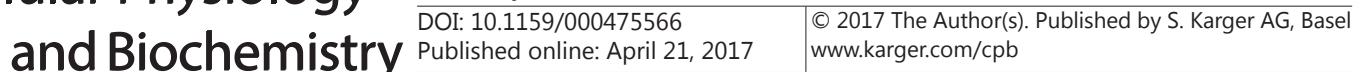

-23 Pihusch R, Salat C, Gohring P, Hentrich M, Wegner H, Pihusch M, Hiller E, Kolb HJ, Ostermann H: Factor xiii activity levels in patients with allogeneic haematopoietic stem cell transplantation and acute graft-versushost disease of the gut. Br J Haematol 2002;117:469-476.

24 Atucha NM, Iyu D, Alcaraz A, Rosa V, Martinez-Prieto C, Ortiz MC, Rosado JA, Garcia-Estan J: Altered calcium signalling in platelets from bile-duct-ligated rats. Clin Sci (Lond) 2007;112:167-174.

-25 Ingeberg S, Jacobsen P, Fischer E, Bentsen KD: Platelet aggregation and release of atp in patients with hepatic cirrhosis. Scand J Gastroenterol 1985;20:285-288.

-26 Pereira J, Accatino L, Pizarro M, Mezzano V, Ibanez A, Mezzano D: In vivo effect of bile salts on platelet aggregation in rats. Thromb Res 1995;80:357-362.

-27 Tag CG, Sauer-Lehnen S, Weiskirchen S, Borkham-Kamphorst E, Tolba RH, Tacke F, Weiskirchen R: Bile duct ligation in mice: Induction of inflammatory liver injury and fibrosis by obstructive cholestasis. J Vis Exp 2015;96:52438.

-28 Lang E, Gatidis S, Freise NF, Bock H, Kubitz R, Lauermann C, Orth HM, Klindt C, Schuier M, Keitel V, Reich M, Liu G, Schmidt S, Xu HC, Qadri SM, Herebian D, Pandyra AA, Mayatepek E, Gulbins E, Lang F, Haussinger D, Lang KS, Foller M, Lang PA: Conjugated bilirubin triggers anemia by inducing erythrocyte death. Hepatology 2015;61:275-284.

29 Elvers M, Herrmann A, Seizer P, Munzer P, Beck S, Schonberger T, Borst O, Martin-Romero FJ, Lang F, May $\mathrm{AE}$, Gawaz M: Intracellular cyclophilin a is an important ca(2+) regulator in platelets and critically involved in arterial thrombus formation. Blood 2012;120:1317-1326.

-30 Fotinos A, Klier M, Gowert NS, Munzer P, Klatt C, Beck S, Borst O, Billuart P, Schaller M, Lang F, Gawaz M, Elvers M: Loss of oligophrenin1 leads to uncontrolled rho activation and increased thrombus formation in mice. J Thromb Haemost 2015;13:619-630.

-31 Jarre A, Gowert NS, Donner L, Munzer P, Klier M, Borst O, Schaller M, Lang F, Korth C, Elvers M: Preactivated blood platelets and a pro-thrombotic phenotype in app23 mice modeling alzheimer's disease. Cell Signal 2014;26:2040-2050.

-32 Walter U, Eigenthaler M, Geiger J, Reinhard M: Role of cyclic nucleotide-dependent protein kinases and their common substrate vasp in the regulation of human platelets. Adv Exp Med Biol 1993;344:237-249.

-33 Michelson AD, Barnard MR: Plasmin-induced redistribution of platelet glycoprotein ib. Blood 1990;76:2005-2010.

34 Pasche B, Ouimet H, Francis S, Loscalzo J: Structural changes in platelet glycoprotein iib/iiia by plasmin: Determinants and functional consequences. Blood 1994;83:404-414.

35 Shiao YJ, Chen JC, Wang CN, Wang CT: The mode of action of primary bile salts on human platelets. Biochim Biophys Acta 1993;1146:282-293.

-36 Reich M, Deutschmann K, Sommerfeld A, Klindt C, Kluge S, Kubitz R, Ullmer C, Knoefel WT, Herebian D, Mayatepek E, Haussinger D, Keitel V: Tgr5 is essential for bile acid-dependent cholangiocyte proliferation in vivo and in vitro. Gut 2016;65:487-501.

-37 Aster RH: Pooling of platelets in the spleen: Role in the pathogenesis of "hypersplenic" thrombocytopenia. J Clin Invest 1966;45:645-657.

-38 Goulis J, Chau TN, Jordan S, Mehta AB, Watkinson A, Rolles K, Burroughs AK: Thrombopoietin concentrations are low in patients with cirrhosis and thrombocytopenia and are restored after orthotopic liver transplantation. Gut 1999;44:754-758.

-39 Peck-Radosavljevic M, Zacherl J, Wichlas M, Sims P, Meng YG, Panzer S, Lipinski E, Steininger R, Muhlbacher F, Pidlich J, Gangl A: Thrombopoietic cytokines and reversal of thrombocytopenia after liver transplantation. Eur J Gastroenterol Hepatol 1999;11:151-156.

40 Leiper K, Croll A, Booth NA, Moore NR, Sinclair T, Bennett B: Tissue plasminogen activator, plasminogen activator inhibitors, and activator-inhibitor complex in liver disease. J Clin Pathol 1994;47:214-217.

41 Wang H, Zhang Y, Heuckeroth RO: Tissue-type plasminogen activator deficiency exacerbates cholestatic liver injury in mice. Hepatology 2007;45:1527-1537.

42 Bergheim I, Guo L, Davis MA, Duveau I, Arteel GE: Critical role of plasminogen activator inhibitor-1 in cholestatic liver injury and fibrosis. J Pharmacol Exp Ther 2006;316:592-600.

43 Wang H, Zhang Y, Heuckeroth RO: Pai-1 deficiency reduces liver fibrosis after bile duct ligation in mice through activation of tpa. FEBS Lett 2007;581:3098-3104.

44 Atucha N, Iyu D, De Rycker M, Soler A, Garcia-Estan J: Altered calcium regulation in freshly isolated aortic smooth muscle cells from bile duct-ligated rats: Role of nitric oxide. Cell Calcium 2003;33:129-135. 


\section{Cellular Physiology Cell Physiol Biochem 2017;41:2133-2149 and Biochemistry Published on/00475566 $2017 \quad \begin{aligned} & \text { DO 2017 The Author(s). Published by S. Karger AG, Basel } \\ & \text { www.karger.com/cpb }\end{aligned}$ \\ Gowert et al.: Platelets and Cholestatic Liver Disease}

45 Atucha NM, Nadal FJ, Alcaraz A, Iyu D, Ortiz MC, Garcia-Estan J: Reduced capacitative calcium entry in the mesenteric vascular bed of bile duct-ligated rats. Eur J Pharmacol 2005;525:117-122.

46 Nadal FJ, Atucha NM, Iyu D, Garcia-Estan J: Interaction of nitric oxide with calcium in the mesenteric bed of bile duct-ligated rats. Br J Pharmacol 2002;135:489-495.

-47 Lang F, Munzer P, Gawaz M, Borst O: Regulation of stim1/orai1-dependent ca2+ signalling in platelets. Thromb Haemost 2013;110:925-930.

-48 Liu G, Chen H, Alzoubi K, Umbach AT, Gawaz M, Stournaras C, Lang F: Rapid upregulation of orai1 abundance in the plasma membrane of platelets following activation with thrombin and collagen related peptide. Cell Physiol Biochem 2015;37:1759-1766.

49 Liu G, Chen H, Borst O, Gawaz M, Vortkamp A, Schreiber R, Kunzelmann K, Lang F: Involvement of ca2+ activated cl- channel ano6 in platelet activation and apoptosis. Cell Physiol Biochem 2015;37:1934-1944.

50 Xia W, Li Y, Wang B, Chen J, Wang X, Sun Q, Sun F, Li Z, Zhao Z: Enhanced store-operated calcium entry in platelets is associated with peripheral artery disease in type 2 diabetes. Cell Physiol Biochem 2015;37:1945-1955.

51 Witters P, Hoylaerts M, Freson K, de Vos R, van Pelt J, Nevens F, van Geet C, Cassiman D: Adp-degrading enzymes inhibit platelet activation in bile duct-ligated rats. J Thromb Haemost 2010;8:360-368.

52 Li C, Jiang X, Yang L, Liu X, Yue S, Li L: Involvement of sphingosine 1-phosphate (sip)/s1p3 signaling in cholestasis-induced liver fibrosis. Am J Pathol 2009;175:1464-1472.

53 Rauch BH: Sphingosine 1-phosphate as a link between blood coagulation and inflammation. Cell Physiol Biochem 2014;34:185-196.

54 Kundur AR, Singh I, Bulmer AC: Bilirubin, platelet activation and heart disease: A missing link to cardiovascular protection in gilbert's syndrome? Atherosclerosis 2015;239:73-84. 